\title{
Une oisiveté dangereuse. Vers une réglementation du "temps libre" dans les armées bourguignonnes sous Charles le Hardi (1471-1477)
}

Quentin Verreycken

Quentin Verreycken : Quentin Verreycken est diplômé en histoire de l’Université catholique de Louvain (2013). Il est également titulaire d'un Master of Medieval and Renaissance Studies à la KU Leuven (2014). Aspirant F.R.S.-FNRS depuis octobre 2014, il est membre du Centre d'histoire du droit et de la justice (UCL) et du Centre de recherches en histoire du droit et des institutions (USL-B). La thèse de doctorat qu'il prépare est une étude comparée des pardons accordés aux soldats dans les Pays-Bas bourguignons, en France et en Angleterre au XV ${ }^{\mathrm{e}}$ siècle. Il a récemment publié sa première monographie : «Pour nous servir en l'armée ». Le gouvernement et le pardon des gens de guerre sous Charles le Téméraire, duc de Bourgogne (1467-1477), Louvain-la-Neuve, Presses universitaires de Louvain, 2014.

DOI: $10.25518 / 1370-2262.297$

\section{Résumé :}

Cet article traite des réformes disciplinaires du duc de Bourgogne Charles le Hardi en matière militaire entre 1471 et 1476 . Plus spécifiquement, on analysera la tentative du duc de réglementer le «temps libre » des soldats bourguignons lorsqu'ils n'étaient pas en train de manœuvrer ou de combattre sur le champ de bataille. Le principal argument défendu ici est qu'en interdisant certaines activité de divertissement, tels les jeux de dé ou les beuveries, et en instituant des entraînements collectifs aléatoires afin de prévenir la désertion et insuffler un esprit de loyauté parmi les gens de guerre, Charles tenta de diffuser un nouveau modèle de discipline militaire dans ses armées ; un modèle qui, en dépit des échecs du duc, peut être considéré comme l'un des points d'origine de la révolution militaire du XVI ${ }^{\mathrm{e}}$ siècle.

Mots-clés : contrôle social, discipline, histoire militaire, lettres de rémission, Pays-Bas bourguignons, révolution militaire

\section{Abstract :}

This paper discusses the duke of Burgundy Charles the Bold's disciplinary reforms on military matters from 1471 to 1476 . More precisely, it focuses on the duke's intent to regulate the "free time" of Burgundian soldiers when they were not maneuvering or fighting at battle. The main argument formulated here is that by prohibiting several spare-time activities such as dice games or binge drinking and by instituting random collective training in order to prevent desertion and inspire loyalty to men of war, Charles tried to spread a new model of military discipline in his armies ; a model which, in spite of the duke's failures, can be considered as a starting point of the sixteenth-century military revolution.

\section{Introduction}

De la guerre à la fin du moyen âge, on imagine d’abord les batailles rangées, les sièges prolongés, 
Une oisiveté dangereuse. Vers une réglementation du "temps libre" dans les ar...

voire éventuellement les ravages infligés à la population. Pourtant, à lire les chroniqueurs du temps, on s'aperçoit rapidement que les combats occupent une place minoritaire dans la vie du soldat, qu'il soit en campagne ou placé en garnison. Force est pourtant de constater qu'à ce jour, les activités quotidiennes de l'homme de guerre du $\mathrm{XIV}^{\mathrm{e}}$ ou du $\mathrm{XV}^{\mathrm{e}}$ siècle, à commencer par ses divertissements, restent encore peu étudiées1, même si les travaux récents de Franck Viltart ou d’Hervé Martin et Marc Russon sur les camps militaires médiévaux fournissent des renseignements précieux sur les occupations des soldats durant les périodes où ils ne combattent pas 2 . Trois raisons peuvent expliquer ce manque d'intérêt de l'historiographie. La première, la plus évidente, concerne les sources disponibles pour une telle étude : il est rare que l'on dispose d'archives militaires régulières pour la fin du moyen âge, en ce compris dans des domaines d'activité pourtant prompts à en produire, tels les finances ou la justice 3 . Quant aux sources narratives, les chroniqueurs et mémorialistes se focalisent généralement sur les grands personnages et évènements plutôt que sur la vie quotidienne des gens de guerre $\underline{4}$. La deuxième raison tient lieu à une certaine préférence de l'histoire militaire récente pour les travaux sur les structures des armées, les profils sociaux des soldats, ou éventuellement les activités illicites commises par ceux-ci, plutôt que sur leur quotidien $\underline{5}$. La dernière est davantage conceptuelle : comment peut-on qualifier et compartimenter le quotidien des soldats dès lors qu'ils ne combattent ou ne manœuvrent pas ? À partir de quel moment considère-t-on que ces derniers sont oisifs ou bénéficient de périodes de "temps libre » où ils peuvent se livrer à des divertissements (on préfèrera ce terme à celui, plus anachronique, de loisir ) ? La vie de camp peut-elle être considérée comme une période de repos, quand bien même les combattants devaient se livrer à une série de tâches plus ou moins obligatoires, tel l'entretien de l'équipement ? Un tour de garde peut-il être assimilé à un temps d'oisiveté, dans la mesure où il force l'homme de guerre à une certaine forme de statisme, parfois comblée par des passe-temps tels les jeux de dés ? S'il peut être très séduisant pour l'historien de tenter de classer les différentes activités du combattant médiéval, la difficulté à laquelle il se heurte témoigne en elle-même d'une époque où la discipline militaire moderne n'est pas encore établie et où l'emploi du temps du soldat n'est pas clairement défini.

Malgré les biais qu'elle introduit, regarder au travers de la lorgnette de l'histoire de la justice et du crime est probablement l'une des façons les plus efficaces d'étudier le quotidien des gens de guerre. Les sources judiciaires telles les lettres de rémission peuvent s'avérer des documents particulièrement précieux qui témoignent de certaines activités de "divertissement » des combattants, comme les jeux de hasard, les beuveries à la taverne, ou les visites auprès des filles de joie. En quelques occasions, les sources narratives peuvent également attester que l'oisiveté des gens de guerre faisait partie des facteurs d'émergence de troubles au sein des armées. La législation militaire, enfin, contient parfois quelques dispositions réglant les activités des soldats à des fins disciplinaires. Ainsi, pour en apprendre davantage sur le quotidien des soldats, il faut paradoxalement s'intéresser aux situations exceptionnelles, lorsque la violence émerge de l'ordinaire, car ce sont ces situations qui ont tendance à faire parler des sources autrement restées muettes. À cet égard, la richesse documentaire qu'offrent les lettres de rémission est aujourd'hui bien connue des historiens qui tentent de reconstituer le quotidien des médiévauxz. Pourtant, elle ne doit pas faire oublier la nature et les circonstances de production des documents qui sont exploités : source judiciaire produite par la chancellerie royale ou princière, la lettre de rémission est un document par lequel le souverain remet et pardonne un crime (généralement un homicide), suspendant les poursuites judiciaires ou l'exécution d'une peineㅇ. Ainsi, le récit présenté dans l'exposé des motifs de la lettre, basé sur la demande de grâce originale introduite par le requérant (appelé dans la source suppliant), s'il contient effectivement toute une série de renseignements 
sur la vie quotidienne, est d'abord destiné à narrer les circonstances qui ont amené le suppliant à commettre un crime. Il en résulte que ce récit n'est en aucun cas un témoignage sans intermédiaires que le suppliant porterait sur lui-même, pour paraphraser la formule qu'employait jadis Emmanuel Le Roy Ladurie pour qualifier le célèbre registre de l'inquisiteur Jacques Fournier $\underline{9}$ : d'une part, le suppliant avait toutes les raisons de tordre les évènements afin de diminuer sa responsabilité et de rendre ses actes conformes aux critères de rémissibilité définis par le souverain et son conseil, et d'autre part, puisque le récit en question est celui d'un crime, il ne relève pas à proprement parler de l'ordinaire, mais plutôt d'une situation où les «apparences normales » sont brisées dès lors que la violence fait irruption, quand bien même cette dernière reste à l'époque bien intégrée dans les rapports sociaux $\underline{10}$.

On considère généralement que $c^{\prime}$ est à partir du $\mathrm{XVI}^{\mathrm{e}}$ siècle que la professionnalisation des armées et l'expansion considérable de leur taille auraient amené à une bureaucratisation des institutions militaires en même temps que des structures de l'État, ceci afin d'assurer un meilleur contrôle des troupes $\underline{11}$. La «révolution militaire », comme l'appellent Michael Roberts et Geoffrey Parker, aurait ainsi conduit au développement de nouveaux dispositifs coercitifs et à l'invention d'une véritable «discipline militaire » moderne12. Certains historiens ont cependant souligné que c'est $\mathrm{au} \mathrm{XV}^{\mathrm{e}}$ siècle que les premières armées professionnelles se mettent en place, tandis que le rôle du soldat depuis la fin de la guerre de Cent ans est progressivement redéfini $\underline{13}$. Andrew Ayton, J.L. Price et Clifford J. Rogers n'hésitent d'ailleurs pas à parler de "révolution militaire médiévale » pour désigner les changements qui s'opèrent à la fin du moyen âge dans les structures des armées14. Parmi les acteurs majeurs de ces bouleversements, on trouve le duc de Bourgogne Charles le Hardi, dit également le Téméraire. Bien que court (1467-1477), son règne donna lieu à plusieurs réformes institutionnelles importantes, dont un profond remaniement (sinon une refonte) des structures militaires en 1471. Si les armées bourguignonnes étaient basées jusque-là sur un mode de recrutement de type féodal (la levée), le duc institua en parallèle des troupes permanentes recrutées sur une base volontaire, les " compagnies d'ordonnance », dont l'organisation s'inspirait à la fois des modèles français et italien $\underline{15}$. Il rénova également la justice militaire en rendant permanent l'office du prévôt des maréchaux, tout en multipliant les dispositions disciplinaires dans son activité législative16. Il usa enfin de son droit de grâce afin de promouvoir parmi les troupes un modèle de comportement basé sur le service du prince17. C'est dire, donc, si Charles le Hardi eut à cœur d'introduire une véritable discipline militaire au sein de ses armées. On pourra bien entendu objecter que de telles innovations furent bien éphémères, étant donné la fin brutale du duc face aux armées de René II de Lorraine devant les murs de Nancy en janvier 1477. La débâcle des armées bourguignonnes fut d'ailleurs suivie par une série de troubles dans les anciens PaysBas qui conduisirent la nouvelle duchesse Marie de Bourgogne à abolir une partie des réformes institutionnelles accomplies par son père. Force est pourtant de constater que si l'entreprise du Téméraire fut brusquement interrompue, celle-ci fut au moins partiellement reprise par ses successeurs durant les décennies suivantes. Ainsi, le Parlement de Malines, la plus haute juridiction des anciens Pays-Bas, établi en 1473 et aboli en 1477, subsistera sous la forme d'un Grand Conseil avant d'être à nouveau fixé officiellement à Malines en 150418. Quant aux compagnies d'ordonnance, on estime que malgré leur échec, elles furent rétablies vers 1478 par Maximilien de Habsbourg, que leurs structures ne furent par la suite revues profondément qu'une seule fois, en 1545, et qu'elles subsistèrent sous cette forme jusqu'à la fin du XVII ${ }^{\mathrm{e}}$ siècle $\underline{19}$.

En dépit des échecs incontestables de Charles le Hardi, ses réformes paraissent avoir eu un impact certain sur le siècle suivant, ne serait-ce que sur le plan institutionnel et militaire. Or, l'un des 
Une oisiveté dangereuse. Vers une réglementation du "temps libre" dans les ar...

aspects les plus originaux des dispositions nouvelles introduites par la législation du Téméraire consiste précisément en une tentative du duc d'assurer une meilleure discipline en luttant contre l'oisiveté des soldats, ceci au travers de l'élaboration d'une réglementation de leur temps libre et de certains de leurs divertissements. Pour saisir la portée et le contexte de cette réglementation, nous examinerons en quoi l'inactivité pouvait être source de désordre au sein des armées, qu'il s'agisse de conflits interpersonnels (bagarres et rixes) ou de troubles collectifs (émeutes et mutineries). Nous verrons ensuite comment le duc de Bourgogne tenta de limiter ces facteurs d'émergence de la violence en proscrivant une série d'activités. Nous analyserons, enfin, les dispositions prises par Charles afin de promouvoir un meilleur comportement des soldats durant les périodes d'inactivité. La documentation exploitée dans le cadre de cette contribution a déjà été évoquée : outre la législation (les ordonnances militaires) de 1471 à $1476 \underline{20}$ et les sources narratives (principalement les œuvres de Jean de Haynin et Jean Molinet, ainsi que la correspondance des ambassadeurs du duc de Milan auprès du Téméraire21), nous analyserons quelques-unes des trois cents lettres de rémission octroyées par le duc de Bourgogne au cours de son règne.

\section{L'oisiveté comme source de sociabilités conflictuelles}

Parmi près de 300 copies de lettres de rémission, abolition et rappel de ban octroyées par Charles le Hardi et conservées dans le fonds des registres de l'audience de la Chambre des comptes de Lille aux Archives départementales du Nord22, nous avons relevé 26 lettres qui ont été octroyées à des gens de guerre alors qu'ils étaient au service du duc dans ses armées $\underline{23}$. Il ressort de l'analyse de ces documents que les actes de violence pardonnée commis par les gens de guerre (tous des cas d'homicide commis en état de légitime défense ou sous la colère) se produisent toujours en dehors du champ de bataille, de préférence durant des périodes de repos. Ainsi, dans $23 \%$ des cas, le crime s'est déroulé au sein du logis d'un homme de guerre. Une cohabitation sur le long terme des combattants pouvait en effet être source de conflits, et le logement, en tant que lieu de sociabilité et d'échange où les soldats s'invitaient les uns les autres pour boire et manger, devenait le théâtre de querelles qui débouchaient parfois sur un crime de sang. Les gens de guerre avaient eux-mêmes conscience de ce danger, comme le suggère la lettre de Jaques Anthoine, dans laquelle il raconte que deux de ses amis, Jaques de Chantemelle et Perrenet Coffineau, avaient refusé son invitation à venir dîner à son logis, doubtant qu'il n'y eust debat24.

Ex aequo avec le logis, la taverne est également le lieu privilégié de conflits dans six autres cas. Les rixes d'auberges font en effet partie des scènes classiques que l'on trouve dans les archives judiciaires de la fin du moyen âge25. Lieu de sociabilité par excellence, la taverne est à la fois un lieu de repos, de passage et de rencontres. En plus de vieilles animosités qui peuvent s'y réveiller, l'alcool et les jeux d'argent favorisent l'émergence de conflits parfois violents. Lorsqu'il est en garnison, l'homme de guerre peut se mêler à la population en rejoignant les établissements locaux. En campagne, durant les longues périodes de cantonnement, des tavernes provisoires étaient également construites dans les camps, comme en témoigne Jean Molinet lorsqu'il décrit le camp bourguignon établi durant le siège de la ville de Neuss (juillet 1474-juin 1475) : il y avoit forges, tavernes, cabarès, baingneries, hostelleryes et brasseries26. Olivier de La Marche, pour sa part, ne dit pas autre chose dans ses mémoires : il y avoit hosteleries, jeux de paulmes et de billes, cabaretz, tavernes et toutes choses que l'on scut demander27. Maintenir un espace de divertissement était indispensable durant les longues périodes d'immobilité pour conserver le moral des troupes $\underline{28}$. Pour Éric Bousmar, certains de ces établissements, à commencer par les baingneries, pouvaient en outre être des lieux de prostitution $\underline{29}$. Quoi qu'il en soit, deux lettres de rémission ont effectivement 
été octroyées pour des homicides commis durant une rixe de taverne dans le camp bourguignon devant le siège de Neuss, en novembre et décembre 1474르. Le récit des évènements suit lui-même toujours un scénario plus ou moins identique : le repas ou la beuverie dégénère au moment où des questions d'argent interviennent, qu'il s'agisse pour les convives de payer l' " escot » (la part de chacun de l'addition) ou de régler le vainqueur d'un jeu de hasard. L'un des participants refuse, prétextant l'un ou l'autre motif, l'honneur de l'un ou de l'autre est mis en doute, et ceci débouche sur une rixe au cours de laquelle un individu est tué

(...) Illec souppans ensemble, lesquelz après ce qu'ilz orent presques souppé, firent une maniere de compte par le quel ilz trouverent quatre patars bons dont par ung commun adviz il achetroit du poisson. Et après qu'il fut mengié, persirent leurdit compte par le quel ilz deurent avec lesdits quatre patars chacun trois gros de notre monnaie de Flandres, que ledit suppliant tira hors de sa bourse, les mist sur la table et furent bailliez a celuy qui avoit baillé et vendu ledit poisson. Et après pluiseurs parolles furent lesdits trois gros demandez par ledit Pierret audit suppliant pour son escot, a quoy il respondit qu'il les avoit mis sur la table avec lesdits quatre patars (...). Lors dist iceluy qu'il comictoit que l'un d'eulx eust prins lesdits trois gros, dont eust despit ledit feu Pierret et dit audit supliant qu'il estoit ung broulleur de cela dire, et ledit supliant luy respondit qu'il avoit menty car il n'estoit point broulleur, lors iceluy feu Pierret tira sa daghe pour frapper iceluy suppliant (...) [et] frappa de sa dite dague ledit suppliant sur la teste et ou bras jusques a grant effusion de sang, quoy veant iceluy suppliant luy donna aussi ung cop de sa daghe ou costé, et quinze jours après ou plus iceluy feu Pierret termina vie par mort $\gg \underline{31}$.

En plus des crimes commis à la taverne ou au logis, les lettres de rémission octroyées par Charles le Hardi offrent encore trois autres exemples d'homicides commis au cours d'une activité de divertissement de soldats. Deux concernent des accidents survenus, l'un durant une représentation théâtrale en ville au cours de laquelle le suppliant fut invité à tirer un coup d'arquebuse, l'autre lors d'une compétition de tir à l'arc entre archers de compagnies militaires urbaines $\underline{32}$. Le troisième cas résulte d'un jeu entre soldats ayant dégénéré33. Au cours du mois de mai 1473, l’homme d'armes Emond de Thubeauvile et ses compagnons, en garnison dans la ville de Gueldre, pour passer le temps se jouoient l'ung avec l'autre. L'un d'eux, surnommé le seigneur de Malgouverne, fist par esbattement audit suppliant desvetir robe, la quelle il donna a Jehan de Nempole, et ce fait rencontra feu Guillement de Senecourt au quel il fist pareillement desvetir sa robe et la donner audit suppliant. Ce dernier, dit par joyeuseté que puis que ladite robe estoit sienne et que ledit de Malgouverne la luy avoit donnee, il vouloit avoir l'une des manches de ladite robe plus avant fendue qu'elle n'estoit. Mais le dénommé Guillement de Senecourt prit mal ce que le suppliant présente comme une plaisanterie et se mit à profferer pluiseurs parolles injurieuses et minatoires. Le suppliant lui répondit qu'il estoit bien folastre de soy courouchier quant on se jouoit alors, en luy disant en oultre qu'il s'en alast et que a luy il ne vouloit point de noise. En réaction, Guillement dégaina une hachette et s'approcha d'Emond qui, sentant sa vie menacée, sortit sa dague et poignarda son adversaire, provoquant sa mort. Le suppliant, visiblement soucieux de se disculper auprès du prince, ajoute dans son récit qu'il n'avait nullement l'intention de tuer sa victime, voulant la blesser à la cuisse, mais qu'il toucha accidentellement l'aine suite à un mouvement brusque de Guillement.

En apparence isolées, ces rixes survenues en période d'inactivité constituaient souvent l'élément déclencheur d'émeutes à grande échelle lorsque des soldats d'origines différentes étaient impliqués. 
Une oisiveté dangereuse. Vers une réglementation du "temps libre" dans les ar...

En effet, les armées bourguignonnes étaient loin de former un ensemble homogène de combattants : comme le résume fort bien l'ambassadeur milanais Francesco Petrasanta, les troupes de Charles le Hardi étaient composées de molti genti d'ogni qualitate (...) et di varj paesi34. À l'inévitable diversité sociale des troupes composées d'hommes d'armes de noble extraction, de fieffés, de miliciens et de mercenaires, s'ajoutait encore la diversité ethnique des gens de guerre issus de pays différents. Non seulement le duc de Bourgogne recrutait des gens de guerre parmi tous ses territoires, mais dès 1473, il commença également à engager massivement des mercenaires lombards et anglais, qu'il intégra dans les compagnies d'ordonnance 35. Or, des tensions pouvaient naître entre soldats de «nationalité » différente qui cohabitaient sur un long terme, ce qui aboutissait à des querelles de longues durées entre groupes ethniques. Antoine d'Appiano, collègue de Petrasanta, rapporte ainsi des évènements survenus le dimanche 21 avril 1476, alors que les armées bourguignonnes sont retranchées au camp de Lausanne après la défaite de Grandson : un soldat lombard fut tué au cours d'une rixe par des Anglais, ce qui provoqua la colère des Italiens, qui organisèrent une expédition punitive au cours de laquelle ils tuèrent sept hommes. Le duc de Bourgogne, apprenant la nouvelle, ordonna d'exécuter les coupables. Mais cette décision ne fit qu'empirer les choses: vers dix heures du soir, les troupes lombardes quittèrent le camp pour se mettre en ordre de bataille face aux Anglais qui avaient déjà pris les armes. Après deux heures d'émeute et l'intervention de Charles qui, bien que souffrant de fièvre, monta à cheval et se rendit sur place pour contenir les Anglais, le tumulte s'acheva vers minuit. Deux jours plus tard, l'ambassadeur milanais rapporta que les Anglais s'en prenaient encore fréquemment aux Lombards, sans distinction de rang36. On pourrait objecter qu'il ne s'agit ici que de rixes entre deux groupes de mercenaires étrangers, ne partageant guère un esprit de cohésion commune, mais il apparaît pourtant que les soldats originaires des principautés des Pays-Bas n'échappaient pas à de tels conflits : déjà en mai 1465, durant la guerre du Bien Public, Jean de Haynin rapportait qu'au moment de se loger dans le village de Monchy-la-Gache (actuellement dans le département de la Somme), il y eut ung tres grant remous entre leur gens, chest asavoir entre les Hainuiers et les Flamens, et y eut ung archier tué, noumé Didier, lequel estoit de Mons en Haynau, et s'en y eut pluseurs bleschies (...)그.

\section{L'oisiveté comme objet de contrôle}

Conscient de la nécessité de renforcer son contrôle sur les comportements de ses soldats afin de prévenir les troubles au sein de ses armées, Charles le Hardi introduisit dès 1473 une série de mesures disciplinaires destinées à limiter ce qu'il identifiait comme des facteurs d'émergence de la violence : la présence de femmes, de jeux d'argent, et, en 1476, d'alcool.

Bien que l'on manque encore de travaux à ce sujet, il est aujourd'hui bien connu des historiens que les armées médiévales n'étaient pas composées que de combattants : elles trainaient derrière elles toute une population que l'on peut désigner par le qualificatif $d^{\prime}$ ' inframilitaire », souvent nécessaire à la logistique et à l'approvisionnement des troupes, comprenant aussi bien des religieux, prestres, marchans, pietons, charetons et autres gens $\underline{38}$, parmi lesquels des femmes, qu'il s'agisse des compagnes des soldats ou de prostituées. Or, à en croire l'ordonnance de SaintMaximin de novembre 1473, cette présence féminine provoquait souvent fois plusieurs grands débats et aultres inconvéniens. C'est pourquoi, pour éviter la multitude des femmes qui suivent les compaignies desdits gens de guerre de l'ordonnance, Charles limita le nombre de femmes à trente par compagnie, avec pour interdictions que les soldats les trengne comme propre. Implicitement, le duc semble ainsi dorénavant interdire à ses gens de guerre d'être accompagnés par leur femme ou leur compagne, restreignant la présence féminine à quelques prostituées par compagnie (une 
trentaine face à un millier d'hommes !) $\underline{39}$.

Dans l'article suivant de l'ordonnance, le duc de Bourgogne insiste sur la nécessité que ses gens de guerre soyent et demeurent en la crainte, révérence et obéissance de Dieu, notre créateur, et se abstiennent de choses illicites et deshonnêtes. C'est pourquoi il leur interdit qu'ils blasphèment le nom de Dieu, ne fassent aucuns aultres villains sermens, et aussi ne tiennent jeux de dez, sous peine d'être punis à l'arbitraire de leur conducteur (terme employé dans les textes pour qualifier les capitaines des compagnies d'ordonnance) $\underline{40}$. Comme nous l'avons mentionné ci-dessus, les jeux de hasard font partie du décor traditionnel de la taverne. La boisson aidant, ils sont une cause fréquente de rixes lorsqu'un joueur faisant mauvaise fortune refuse de reconnaître sa défaite ou de payer son dû. Activité oisive par excellence, fréquemment condamnée par les législateurs civils ou ecclésiastiques, les jeux de hasard conduisent également au blasphème, le perdant exprimant son mécontentement en reniant Dieu41. Jean Molinet souligne également l'aspect sacrilège des jeux de dés dans un passage de ses chroniques, à propos du siège de Neuss, où il décrit comment les soldats bourguignons furent logés dans l'abbaye voisine: Les chambres de devotion furent changies en derision; là où l'en soloit estudier enseignements beaulx et notables, on tenoit escole de jeux de déz et de tables.42 Ceci explique l'association, dans la législation du Hardi, entre les jeux de hasard et le blasphème, que le duc condamne à la fois sur les plans disciplinaire et religieux. Par cette interdiction, Charles n'entend pas que réglementer les activités de loisir des gens de guerre, mais également agir sur le plan moral, ce qui est confirmé par la suite dans l'ordonnance de Lausanne de mai 1476, qui durcit et précise les mesures à l'égard des blasphémateurs : ceuxci devaient désormais être exposés durant un jour et une nuit attachés à une roue de chariot et nourris uniquement au pain et à l'eau. En outre, les récidivistes devaient être livrés au prévôt

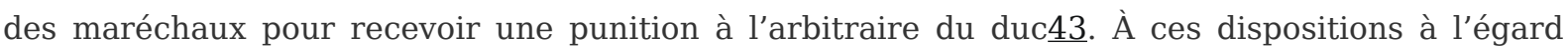
des blasphémateurs s'ajoute également un renforcement général de la discipline militaire, Charles généralisant la peine de mort à l'égard des déserteurs, pilleurs, violeurs et sacrilèges $\underline{44}$. Les putains et ribaudes devaient désormais être chassées des armées, tandis que les conducteurs étaient tenus de réduire la consommation d'alcool de leurs soldats, faisant à leurs gens boire plus d'eaue afin qu'ilz soient moins chaulx durant ledit voyage sur ce mestier $\underline{45}$. Enfin, afin d'assurer la bonne application de sa législation, le duc institua une procédure de dénonciation des chefs de guerre laxistes, promettant par ailleurs que ces derniers subiraient les mêmes peines que les soldats qu'ils étaient censés poursuivre $\underline{46}$.

\section{L'oisiveté comme espace de contrôle et de moralisation}

Si l'on retiendra de l'ordonnance de Lausanne de mai 1476 qu'elle renforça considérablement la coercition à l'égard des gens de guerre, elle demeure avant tout un prolongement de l'ordonnance de Saint-Maximin de novembre 1473. Si l'historien Ferdinand Lot qualifie cette dernière du "plus beau code militaire du siècle $\$ \underline{47}$, c'est en raison des révisions qu'elle apporta de l'organisation des compagnies d'ordonnance, mais également des dispositifs disciplinaires nouveaux qu'elle introduisit. Charles tenta de mettre en place avec ce texte un dispositif poussé d'entraînement des troupes. Il ordonna en effet que ses conducteurs meynent aucune fois, partie de leurs hommes d'armes jouer au champs afin de les entraîner à courre a lance, eux tenir en la courant, joints et serrez, et aussi courre vivement, garder leurs enseignes, eux départir, s'il leur est ordonné et eux rallier en secourant l'un l'aultre par commandement, et la manière de soutenir une charge. Ces entraînements n'étaient pas limités aux hommes d'armes, puisque le texte comporte également des instructions similaires pour les archers et les piquiers. Le but de ces dispositions n'était pas 
Une oisiveté dangereuse. Vers une réglementation du "temps libre" dans les ar...

uniquement de faire des soldats bourguignons de meilleurs combattants, mais également d'assurer un plus grand contrôle de ceux-ci en renforçant l'emprise des autorités militaires sur eux durant les périodes d'inactivité : et ce faisant, lesdits conductiers auront journellement la vue et le regard de leurs gens, lesquels, en ce cas, ne se oseront absenter ni estre dépourvus de leurs chevaulx et harnais, parce qu'ils ne seront pas sûrs du jour que lesdits conductiers les voudront mener audit exercice. Enfin, en mettant ses soldats en ordre et discipline, Charles entendait que ses conducteurs leur apprennent l'amour et obéissance qu'ils doibvent à avoir envers mondit seigneur et à l'exaltation de sa maison, et aussi leur propre honneur et renommée, qui consiste en ce que mondit seigneur, par le moyen de leur bon service, puisse parvenir au reboutement de ses ennemis $\underline{48}$. À cet égard, Richard Vaughan n'a pas tort lorsqu'il qualifie de «drill » ces entraînements dirigés par les conducteurs $\underline{49}$.

Il est généralement considéré par les historiens que le drill, cette discipline militaire censée assurer la cohésion des troupes sur le champ de bataille tout en favorisant un usage optimal de leurs armes, fut introduit dans les armées européennes au début des temps modernes, lorsque, selon les mots de William McNeill, « infantry regained importance in battle as part of combined arms deployment in which cavalry, artillery, and infantry each played a role $" \underline{50}$. À la suite des travaux de Michael Roberts et Geoffrey Parker, il est admis que Maurice de Nassau fut l'un, sinon le premier chef de guerre à élaborer un programme complet de drill à la fin des années 1590. Dans son récent ouvrage consacré aux armées de la République protestante, Olaf van Nimwegen décrit les circonstances et le contenu de cette réforme disciplinaire, rendue nécessaire par la confrontation avec les armées espagnoles, toujours en supériorité numérique $\underline{51}$. L'attention portée à l'instruction des troupes (au travers de l'apprentissage d'une série de séquences de mouvements afin de former les rangs, tirer et recharger, brandir une pique, etc.) et à l'organisation des bataillons, est certes bien plus aboutie que les quelques dispositions introduites par Charles le Hardi en 1473, mais il n'empêche que ces dernières témoignent des préoccupations disciplinaires du duc de Bourgogne. Elles n'ont finalement rien d'étonnant si l'on considère l'influence qu'eut sur Charles le De re militari de Végèce, lequel inspira également Maurice de Nassau⒉ Ainsi, dans une certaine mesure, le souci qui se manifesta dans les Provinces-Unies au début du XVII ${ }^{\mathrm{e}}$ siècle de créer des armées morales en accord avec la doctrine néo-stoïcienne promouvant la vertu individuelle du soldat et la défense du pays plutôt que l'honneur guerrier $\underline{53}$, trouva un précédent, du moins sur papier, dans les ordonnances de Charles le Hardi.

Comme le remarque en effet Christopher Allmand, le duc de Bourgogne insiste dans l'ordonnance militaire d'Abbeville de juillet 1471 sur le fait que ses compagnies d'ordonnance devaient être assemblées pour le bien, seurte \& deffence de nos pays, seigneuries et subjects; \& affin de prestement les preserver \& garder de dommages \& invahissemens que nos ennemis, adversaires \& malveillans y vouldroient faire, justifiant ainsi la mise sur pied d'une force militaire par la défense de la res publica $\underline{54}$. Selon Allmand, cette même ordonnance confirme l'objectif de disciplinarisation des armées bourguignonnes lorsqu'il est précisé que les soldats devront être rassemblés à une certaine date en étant ydoines \& souffisans $\underline{55}$ : en effet, le mot ydoine correspondrait étymologiquement au latin idoneus, soit le terme employé par Végèce pour désigner les troupes choisies pour le service après avoir été testées $\underline{56}$. L'année suivante, l'ordonnance militaire de Bohain de novembre 1472 imposa que les soldats passés à montre prêtent serment d'estre bons et loïaux à mondict seigneur, et qu'ilz le serviront envers et contre tous57. Dans une précédente publication, nous avions sur ce point émis l'hypothèse que l'interdiction par l'ordonnance de Saint-Maximin de novembre 1473 des blasphèmes et villains sermens pouvait être interprétée comme un renforcement supplémentaire 
de la discipline militaire, toute infraction grave à l'autorité princière entraînant par là même un bris de serment $\underline{58}$. Enfin, l'ordonnance de Saint-Maximin de novembre 1473précise le «profil » que devaient rechercher les conducteurs : des gens experts en guerre et de bonne part $\underline{59}$. À lire le dictionnaire de Godefroy, les termes de bonne part renvoient essentiellement à l'extraction sociale noble de l'individu $\underline{60}$, mais puisque les armées bourguignonnes étaient loin d'être exclusivement composées de gentilshommes, on peut raisonnablement les interpréter ici au sens de «moralité » ou de « réputation».

Ces différents éléments suggèrent que la tentative de Charles de règlementer les périodes d'inactivité des gens de guerre faisait partie d'une politique disciplinaire plus large destinée à modeler le comportement des soldats, à imposer une «normalisation disciplinaire » $\underline{61}$ semblable à celle que connaîtront les armées modernes durant la révolution militaire du $\mathrm{XVI}^{\mathrm{e}}$ siècle. En considérant l'armée médiévale comme un milieu à part, à l'écart du reste de la population, on pourrait même être tenté de faire appel au concept d' «institution totale » («total institution ») développé par le sociologue Erving Goffman, c'est-à-dire le «lieu de résidence et de travail [...] où un grand nombre d'individus, placés dans la même situation, coupés du monde extérieur pour une période relativement longue, mènent ensemble une vie recluse dont les modalités sont minutieusement réglées » $\underline{62}$. Au départ des règlements militaires élaborés par Charles le Hardi, peut-on qualifier les armées bourguignonnes d'« institutions totales », et les soldats bourguignons, d'individus totalement disciplinés ? Il convient évidemment de nuancer les déclarations précédentes en les confrontant aux réalités de la vie militaire. En dépit du caractère innovant de l'ordonnance de Saint-Maximin de novembre 1473, celle-ci est loin de régler chaque aspect du temps et de la vie de l'homme de guerre. Quant à l'ordonnance de Lausanne de mai 1476, elle est d'abord une réponse aux émeutes entre Anglais et Lombards ayant eu lieu au mois d'avril, ce qui souligne déjà à quel point les armées du duc de Bourgogne pouvaient être sujettes aux troubles. L'interdiction répétée de divers comportements - la désertion, bien sûr, mais également l'usage d'alcool, les jeux de dés, le blasphème ou la prostitution -, si elle traduit la tentative de mise en place d'un plus grand encadrement disciplinaire des soldats, souligne également les difficultés à faire appliquer ces mesures, comme en témoigne dans l'ordonnance de Bohain de novembre 1472cette accusation faite par Charles à ses conducteurs d'être «mols et lasches à faire les pugnitions »63, reproche réitéré l'année suivante dans l'ordonnance de Saint-Maximin $\underline{64}$.

Les conditions du recrutement des gens de guerre démontrent également que ces derniers étaient loin de correspondre à l'idéal du bon soldat voulu par le duct65. En dépit de l'exigence affichée par Charles de ne recruter que des individus de bonne réputation, il semble dans les faits peu probable que les conducteurs aient véritablement eu en leur possession les moyens de contrôler efficacement la bonne fame et renommée des recrues, ne serait-ce que parce que l'identité d'un individu restait alors fortement attachée au lieu de domicile : pour peu que la recrue fût nouvelle dans la région, il n’y avait guère de moyens pour les autorités de contrôler son passé66. Or, comme en témoignent les lettres de rémission, il était justement d'usage pour les individus s'étant rendus coupables d'un crime d'échapper à la justice locale en fuyant sa juridiction pour se réfugier dans d'estranges marches et contrees. Les armées constituaient un refuge pour les fugitifs qui, contraints de quitter leur région et leur lieu de travail, refaisaient leur vie en choisissant le métier des armes, comme l'attestent une vingtaine de lettres de rémission octroyées par le duc de Bourgogne à des individus ayant rejoint les armées après la commission d'un homicide67. Parmi celles-ci, on trouve la rémission octroyée à Rolandin Le Grain et Pierrot Blancart (août 1474), qui nous apprend l'existence de letres patentes qui ont esté publiees en noz villes et pays de par de 
Une oisiveté dangereuse. Vers une réglementation du "temps libre" dans les ar...

ça ayans rappellez tous banniz et homicides qui nous vouldroient venir servir68. Il est cependant difficile d'évaluer la portée de ces mandements, dans la mesure où nous n'avons pu identifier dans les registres de l'audience allant de 1474 à 1476 «que» 13 lettres de rémission effectivement octroyées à des fugitifs devenus soldats (ce qui constitue tout de même $65 \%$ des cas observés pour l'ensemble du règne du Hardi). Quoi qu'il en soit, ces dispositions attestent du décalage qui existait entre les normes contenues dans la législation militaire et les pratiques concrètes de recrutement : en dépit des déclarations de Charles, la bonne moralité ne semble pas avoir été un critère déterminant de sélection des recrues, étant donné qu'un certain nombre d'entre elles s'étaient déjà rendues coupables d'un crime. On sait par ailleurs quelles difficultés connaîtra le duc tout au long de son règne pour obtenir de ses sujets les ressources financières et humaines dont il avait besoin : il est ainsi probable que la mesure prise par Charles de proposer le pardon en échange du service de guerre était destinée à pallier les difficultés rencontrées à trouver de nouvelles recrues.

Face aux problèmes de recrutement, engager des hors-la-loi ne fut pas la seule option retenue par Charles le Hardi : comme il a déjà été signalé, dès 1473, celui-ci commença à faire un usage massif de mercenaires lombards et anglais, qu'il intégra dans ses compagnies d'ordonnance $\underline{69}$. Dans sa Chronique, l'indiciaire Jean Molinet souligne pourtant qu'il s'agissait là d'une entorse à la doctrine de Végèce :

\footnotetext{
Vegèce conseille aux princes que mieulx vault enseignier ses propres chevaliers au très noble mestier d'armes que prendre estrangiers à sauldées. Et le duc, parpayant ses deniers, estoit servi de Lombars et Englois qui grandement s'employèrent; mais, pour ce qu'il estoit cremu et redoubté de touttes nations et que le ciel et la terre lui favorisoyent plus que à nul aultre, il avoit ce previlège de trespasser les commandemens des philozophes $\underline{70}$.
}

Serait-ce ici le constat de l'échec du duc de Bourgogne à mettre sur pied une armée unifiée ? C'est tout du moins l'avis de Philippe Contamine, qui voit en cet usage massif de mercenaires étrangers le signe de la méfiance de Charles envers ses propres sujets. Pour l'historien français, "Charles a voulu créer non pas une armée bourguignonne (...) qui aurait été l'expression d'un État unifié, mais un instrument militaire à son entière disposition pour la poursuite de ses ambitions », mais il rappelle également que « de toute façon, bien des armées, à l'époque et encore plus tard, n'étaient nullement conçues comme devant favoriser l'émergence d'une identité nationale, elles n'étaient pas un facteur d'intégration $\gg \underline{71}$.

\section{Conclusion}

De 1473 à 1476, la proscription d'une série d'activités et de divertissements durant le temps libre des gens de guerre, suivie par l'introduction d'un entraînement spécifique censé décourager les déserteurs et favoriser l'esprit de cohésion, témoignent de la volonté du duc de Bourgogne de renforcer son emprise sur le corps et le temps de ses soldats, ceci afin d'améliorer leur discipline. Mais le programme ambitieux de Charles le Hardi, qui à bien des égards apparaît comme l'un des points de départs du processus qui mènera à la révolution militaire du $\mathrm{XVI}^{\mathrm{e}}$ siècle, ne doit pas faire oublier les réalités du terrain. Malgré de successifs remaniements, les armées bourguignonnes ont toujours souffert d'indiscipline et d'un manque de cohésion dû à l'usage combiné de mercenaires et de combattants bourguignons aux multiples «nationalités ». Si l'on peut raisonnablement et dans certaines limites voir en Charles un disciple de Végèce et un prédécesseur de Maurice de Nassau, 
si les intentions de réforme et de moralisation des armées qui prendront tout leur sens aux siècles suivants sont bien présentes dans les ordonnances militaires du duc de Bourgogne, Charles le Hardi ne parvint pas à mettre sur pied l'armée parfaitement disciplinée dont il rêvait.

\section{Notes}

1 A contrario, les « jeux militaires » organisés par les compagnies urbaines d'archers ou d'arbalétriers, pour autant que l'on puisse les considérer comme des divertissements, ont déjà fait l'objet de divers travaux : L. CROMBIE, Honor, community and hierarchy in the feasts of the archery and crossbow guilds of Bruges, 1445-81, dans Journal of Medieval History, t. 37, 2011, p. 102-113 ; C. LAMARRE, Les jeux militaires au XVIII ${ }^{e}$ siècle, dans Histoire urbaine, t. 1, 2002, $\mathrm{n}^{\circ} 5, \mathrm{p} .85-103$.

2 F. ViLTART, Le camp à la fin du Moyen Âge. Étude sur l'organisation, les fonctions, la représentation des campements militaires dans l'espace bourguignon (v.1400-v.1500), Thèse de doctorat en histoire inédite, Lille, Université de Lille 3 Charles-de-Gaulle, 2010 ; H. MARTIN et M. Russon, Vivre sous la tente au Moyen Âge, Rennes, Éditions Ouest-France, 2010, 408 p.

3 Pour le cas des anciens Pays-Bas, voir P.-L. GARNIER, Les services de la Trésorerie des guerres et de la Recette de l'artillerie de Charles le Téméraire, dans Revue du Nord, t. 79, 1997, p. 969-991 ; L. VAN MEERBEECK, Inventaire des archives des tribunaux militaires, Gembloux, Duculot, 1939, $64 \mathrm{p}$.

4 Comme le montre É. BOUSMAR, Les rapports hommes/femmes dans les Pays-Bas bourguignons (ca.1440 - ca.1510) : aspects anthropologiques, culturels et politiques, Thèse de doctorat en histoire inédite, Louvain-la-Neuve, Université catholique de Louvain, 1997, ces œuvres contiennent souvent de façon éparse des éléments d'information renseignant sur les conditions de vie en temps de guerre.

5 P. Contamine, Guerre, État et Société à la fin du Moyen Âge. Etudes sur les armées des rois de France. 1337-1494, Paris-La Haye, Mouton, 1972, xxxviii-757 p. (Civilisations et sociétés, 24) ; F. GONZALEZ DE LEON, The road to Rocroi : class, culture and command in the Spanish army of Flanders, 1567-1659, Leyde, Brill, 2008, xvi-406 p. (History of warfare, 52) ; V. TouREILLE, De la guerre au brigandage : les soldats de la guerre de Cent Ans ou l'impossible retour, dans Sorties de guerre. Cahiers du Centre d'études d'histoire de la Défense, t. 24, 2005, p. 29-42 ; ID., Vol et brigandage au Moyen Âge, Paris, PUF, 2006, 310 p. (Le nœud gordien).

6 Comme le rappellent H. MARTin et M. Russon, Vivre sous la tente..., p. 15 : « ce concept [de loisir] est totalement inadéquat pour le Moyen Âge, où seule une étroite couche de dominants disposait réellement de loisirs prolongés, alors que la grande masse de la population ne bénéficiait que de distractions aussi brèves qu'épisodiques ».

7 On trouve déjà au XIX ${ }^{\mathrm{e}}$ siècle une étude de C. DEHAISNES, Étude sur les registres des chartes de l'Audience conservées dans l'ancienne Chambre des Comptes de Lille. Guerres et pillages, crimes et malheurs, mœurs et malheurs dans les Pays Bas du XIV et du XV siècle, dans Mémoires de la Société des sciences, de l'agriculture et des arts de Lille, $4^{\mathrm{e}}$ série, t. I, 1876, p. 329-422, se basant sur les registres de l'audience de la Chambre des comptes de Lille, riches en lettres de rémission bourguignonnes. Près d'un siècle plus tard, Roger Vaultier réalisait son étude demeurée 
Une oisiveté dangereuse. Vers une réglementation du "temps libre" dans les ar...

classique : R. VAULTIER, Le folklore pendant la guerre de Cent ans d'après les lettres de rémission du Trésor des chartes, Paris, Librairie Guénédaud, 1965, xxiii-243 p. Pierre Charbonnier, après s'être intéressé à la situation des jeunes adultes ainsi qu'à la journée du « français moyen » au $\mathrm{XV}^{\mathrm{e}}$ siècle, a réalisé une série d'études sur des localités françaises de la fin du moyen âge, toujours en prenant comme point de départ les lettres de rémission : P. CHARBONNIER, L'entrée dans la vie au $X V^{e}$ siècle, d'après les lettres de rémission, dans Actes des congrès de la SHMESP. $12^{e}$ congrès, Nancy, 1981. Les entrées dans la vie. Initiations et apprentissages, Nancy, Presses universitaires, 1982, p. 71-103 ; ID., Le temps vécu ou la journée du français moyen au XV siècle d'après les lettres de rémission, dans Temps social, temps vécu. Actes du $129^{e}$ congrès national des sociétés historiques et scientifiques, Besançon, 2004, éd. C. MAZAURIC, Paris, CTHS, 2007, p. 361-370 ;ID., Aigueperse et ses campagnes à la fin du Moyen Age d'après les lettres de rémission, dans Sparsae, t. 62, 2008, p. 31-51 ; ID., Montluçon et sa région au XV siècle d'après les lettres de rémission, dans Bulletin de la Société des amis de Montluçon, t. 56, 2005, p. 55-86.

8 Voir également, pour la France, la définition classique de C. GAUVARD, « De grace especial ». Crime, état et société en France à la fin du Moyen Âge, $2^{\mathrm{e}}$ éd., Paris, Publications de la Sorbonne, 2010, p. 61 (Les classiques de la Sorbonne, 1) : « La lettre de rémission est un acte de la Chancellerie par lequel le roi octroie son pardon à la suite d'un crime ou d'un délit, arrêtant ainsi le cours ordinaire de la justice, qu'elle soit royale, seigneuriale, urbaine ou ecclésiastique ».

9 E. Le Roy Ladurie, Montaillou, village occitan de 1294 à 1324, $2^{\mathrm{e}}$ éd., Paris, Gallimard, 1982, p. 9.

10 E. Goffman, The presentation of self in everyday life, New York, Doubleday, 1959, 259 p. (Anchor Books). Sur la place de la violence dans la société médiévale, on lira notamment G. SCHWERHOFF, Justice et honneur. Interpréter la violence à Cologne (XV ${ }^{e}-X V I I I^{e}$ siècle), dans Annales. Histoire, Sciences sociales, t. 62, 2007, n 5, p. 1031-1061.

11 S. Gunn, D. GrummitT et H. Cools, War, state, and society in England and the Netherlands, 1477-1559, Oxford, Oxford University Press, 2007, xiv-395 p.

12 G. PARKeR, La révolution militaire. La guerre et l'essor de l'Occident, 1500-1800, Paris, Gallimard, 1993, 276 p. ; M. ROBERTs, The military revolution, 1560-1660, dans The military revolution debate : reading on the military transformation of early modern Europe, éd. C. J. ROGERS, Boulder, Westview, 1995, p. 13-35.

13 C. T. Allmand, Changing views of the soldier in late medieval France, dans Guerre et société en France, en Angleterre et en Bourgogne, $X I V^{e}-X V^{e}$ siècle, éd. P. CONTAMINE et C. GIRY-DELOISON, Lille, Université Charles de Gaulle. Centre d'histoire de la région du Nord et de l'Europe du NordOuest, 1991, p. 171-188. Voir aussi A. AYTON et J.L. PRICE, Introduction : the military revolution from a medieval perspective, dans The medieval military revolution : state, society and military change in medieval and early modern Europe, éd. A. AYTON et J.L. PRICE, Londres, Tauris, 1995, p. 10.

14 Ibidem; C. J. ROgERS, The military revolutions of the Hundred Years War, dans The military revolution debate..., éd. C. J. RogeRs, Boulder, Westview, 1995, p. 55-95. 
15 Les armées de Charles le Hardi dans leur ensemble ont fait l'objet de plusieurs études, dont certaines déjà anciennes : C. BRUSTEN, L'armée bourguignonne de 1465 à 1468, Bruxelles, Éditions Fr. van Muysewinkel, 1953, xxxvii-275 p. ; ID., L'armée bourguignonne de 1465 à 1477, dans Revue internationale d'histoire militaire, t. 20, 1959, p. 452-466 ; H. L. G. GUILLAUME, Histoire de l'organisation militaire sous les ducs de Bourgogne, Bruxelles, Hayez, 1848, 204 p. (Mémoires couronnés et mémoires des savants étrangers, publiés par l'ARSLBA de Belgique, 22) ; J. de LA Chauvelays, Les armées des trois premiers ducs de Bourgogne de la Maison de Valois, dans Mémoires de l'Académie des sciences, arts et belles-lettres de Dijon, $3^{\mathrm{e}}$ série, t. 6, 1880 , p. 18-335 ; ID., Mémoires sur la composition des armées de Charles le Téméraire dans les deux Bourgognes, dans Mémoires de l'Académie des sciences, arts et belles-lettres de Dijon, $3^{\mathrm{e}}$ série, t. 5, 1878-1879, p. 138-369 ; B. SCHNERB, L'État bourguignon 1363-1477, Paris, Perrin, 1999, p. 262- 274 ; R. VAughan, Charles the Bold, the last Valois duke of Burgundy, Londres, Longman, 1973, p. 197-229. Sur les compagnies d'ordonnance, on consultera plus spécifiquement C. BRUSTEN, Les compagnies d'ordonnance dans l'armée bourguignonne, dans Grandson 1476. Essai d'approche pluridisciplinaire d'une action militaire $d u X V^{e}$ siècle, Lausanne, Centre d'histoire, 1976, p. 112-169 ; ID., La fin des compagnies d'ordonnance de Charles le Téméraire, dans Cinqcentième anniversaire de la bataille de Nancy (1477). Actes du Colloque organisé par l'Institut de recherche régionale en sciences sociales, humaines et économiques de l'université de Nancy II (Nancy, 22-24 septembre 1977), Nancy, Université de Nancy II, 1978, p. 363-375 ; H. L. G. Guillaume, Histoire des bandes d'ordonnance des Pays-Bas, Bruxelles, Hayez, 1873, 248 p. (Mémoires de l'Académie royale des sciences, des lettres et des beaux-arts de Belgique, 40). Sur le système féodal de recrutement, que Charles ne manqua pas également de réformer, voir $\mathrm{C}$. LEROUX, Guerre et enquêtes féodales sous Charles le Téméraire, dans Revue du Nord, t. 402, 2013, n 3, p. 859-880 ; B. SCHNERB, Le recrutement social et géographique des armées des ducs de Bourgogne (1340-1477), dans Guerre, pouvoir, principauté, éd. J.-M. CAUCHIEs, Bruxelles, FUSL, 2002, p. 53-67 (Cahiers du CRHiDI, 18) ; ID., L'État bourguignon..., p. 271-272.

16 B. SCHNERB, "L'honneur de la maréchaussée ». Maréchalat et maréchaux en Bourgogne des origines à la fin du XV $V^{e}$ siècle, Turnhout, Brepols, 2000, p. 155-175; voir aussi ID., Un thème de recherche : l'exercice de la justice dans les armées des ducs de Bourgogne (fin XIV - fin XV $V^{e}$.), dans Publications du Centre européen d'études bourguignonnes, t. 30, 1990, p. 109-112 ainsi que J.-M. CAUCHIES, La désertion dans les armées bourguignonnes de 1465 à 1476, dans Revue belge $d^{\prime}$ histoire militaire, t. 22, 1977, $\mathrm{n}^{\circ}$ 2, p. 132-148.

17 Q. VERREYCKEN, «Pour nous servir en l'armée ». Le gouvernement et le pardon des gens de guerre sous Charles le Téméraire, duc de Bourgogne (1467-1477), Louvain-la-Neuve, Presses universitaires de Louvain, 2014, 322 p. (Histoire, justice, sociétés).

18 J.-M. CAUchies, Philippe le Beau, le dernier duc de Bourgogne, Turnhout, Brepols, p. 70. Voir également L. T. MAES, Het Parlement en de Grote Raad van Mechelen : 1473-1797, Anvers, Uitgeverij C. de Vries-Brouwers, 2009, 248 p.

19 H. L. G. Guillaume, Histoire des bandes d'ordonnance..., p. 412.

20 Ordonnance d'Abbeville du 31 juillet 1471, éditée dans Mémoires pour servir à l'histoire de France et de Bourgogne, contenant un journal de Paris sous les règnes de Charles VI et de Charles VII, vol. 2, Paris, 1729, p. 286-293 ; ordonnance de Bohain du 13 novembre 1472, éditée par L. 
Une oisiveté dangereuse. Vers une réglementation du "temps libre" dans les ar...

Gollut, Les mémoires historiques de la république séquanoise et des princes de la Franche-Comté de Bourgogne, éd. C. Duvernoy et E. Bousson DE MAIRET, nouv. éd., Arbois, Auguste Javel, 1846, col. 1246-1257 ; ordonnance de Saint-Maximin de novembre 1473, éditée par H. L. G. GuILLAUME, Histoire de l'organisation militaire..., p. 191-202 ; et celle de Lausanne de mai 1476, éditée par J. de LA ChAUvelays, Les armées des trois premiers ducs de Bourgogne..., p. 314-333. Il existe également une édition en italien dans les Dépêches des ambassadeurs milanais sur les campagnes de Charles-le-Hardi, duc de Bourgogne, de 1474 à 1477, éd. F. de Gingins LA SARRA, vol. 2, Paris, Cherbuliez, 1858, p. 152-174.

21 J. DE HAYnin, Mémoires de Jean, Sire de Haynin et de Louvignies 1465-1477, éd. D. Brouwers, nouv. éd.,, Liège, Chez Denis Cormaux, 1905, 2 vol. (Société des Bibliophiles Liégeois. Publications in-8 ${ }^{\circ}$ 37-38) ; J. Molinet, Chroniques de Jean Molinet (1474-1506), éd. G. Doutrepont ET O. Jodogne, Bruxelles, Palais des Académies, 1935-1937, 3 vol. ; Dépêches des ambassadeurs milanais...

$\underline{22}$ ADN B 1693, 1694, 1695 et 1698.

$23 \mathrm{Au}$ total, ce sont environ 70 lettres de rémission qui ont été octroyées à des individus ayant commis un crime avant, pendant ou après qu'ils aient servi le duc dans ses armées. Pour plus de détails, voir Q. VERREYCKEN, « Pour nous servir en l'armée »...

$\underline{24}$ ADN B $1693 f^{\circ} 51 r^{\circ}-52 v^{\circ}$.

25 M. Bourin et B. CHEVALIER, Le comportement criminel dans les pays de la Loire moyenne, d'après les lettres de rémission (vers 1380 - vers 1450), dans Annales de Bretagne et des pays de l'ouest, t. 88, 1981, n 3 : Criminalité et répression (XIV -XIX ${ }^{e}$ siècles), p. 245-263; K. TROCH, «Plein comme un pot! » Les cabarets et la boisson à Namur durant la première moitié du XVIII siècle, dans Buveurs, voleuses, insensés et prisonniers à Namur au $18^{e}$ siècle : Déviance, justice et régulation sociale au temps des Lumières, éd. I. PARMENTIER, X. ROUSSEAUX ET S. AusPeRT, Namur, Presses Universitaires de Namur, 2012, p. 14-46 (Histoire, Art et Archéologie, 14).

$\underline{26}$ J. Molinet, Chroniques..., vol. 1, p. 57.

27 O. de La Marche, Mémoires d'Olivier de la Marche, éd. Henri Beaune et J. d'Arbaumont, t. 3, Paris, Renouard, 1888 (Société de l’Histoire de France, 72), p. 92.

28 H. MARTin et M. Russon, Vivre sous la tente..., p. 233.

29 É. BousmaR, Les rapports entre hommes et femmes au 15e s. : courtoisie et réalité vécue. Une étude d'après les chroniques de M. d'Escouchy, J. du Clercq, O. de la Marche et J. de Haynin, Mémoire de licence en histoire inédit, Louvain-la-Neuve, Université catholique de Louvain, 1991, p. 250.

30 ADN B $1695 f^{\circ} 72 \mathrm{r}^{\circ}-\mathrm{v}^{\circ}, 75 \mathrm{r}^{\circ}$.

$\underline{31}$ ADN B $1695 \mathrm{f}^{\circ} 72 \mathrm{r}^{\circ}-\mathrm{v}^{\circ}$.

32 ADN B $1695 \mathrm{f}^{\circ} 36 \mathrm{r}^{\circ}-\mathrm{v}^{\circ}, 69 \mathrm{v}^{\circ}-70 \mathrm{r}^{\circ}$.

$\underline{33}$ ADN B $1695 \mathrm{f}^{\circ} 2 \mathrm{v}^{\circ}-3 \mathrm{r}^{\circ}$. 
34 Dépêches des ambassadeurs milanais..., vol. 2, lettre CLII, p. 23.

35 L.-É. RoulET, Présence et engagement des combattants anglais à Grandson et à Morat, dans Publications du Centre européen d'études bourguignonnes, t. 35, 1995, p. 107-122 ; B. SCHNERB, Troylo da Rossano et les Italiens au service de Charles le Téméraire. Avec deux pièces justificatives, dans Francia, t. 26, 1999, n² 1, p. 103-128 ; G. SolDI-RondININI, Condottieri italiens au service de Charles le Hardi, pendant les guerres de Suisse (1474-1477), dans Publications du Centre européen d'études bourguignonnes, t. 20, 1980, p. 55-62.

36 Dépêches des ambassadeurs milanais..., vol. 2, lettres CLXXIX p. 83-87 et CLXXX p. 87-92. Voir également L.-É. RoulET, Présence et engagement..., p. 107-109.

37 J. de HaYnin, Mémoires de Jean..., vol. 1, p. 24-25.

$\underline{38}$ Ibid., p. 213.

39 H. L. G. Gulllaume, Histoire de l'organisation militaire..., p. 201.

40 Ibidem.

41 J.-M. MEHL, Les jeux de dés au XV siècle d'après les lettres de rémission, dans Les jeux à la Renaissance, éd. P. ARIÈs et J.-C. MARgolin, Paris, Vrin, 1982 (De Pétrarque à Descartes, 43), p. 625 et 630 ; J. VAN HUMBEECK, Exploitation et répression des jeux de d'argent en Flandre aux XIVe et XVe siècles, dans Tijdschrift voor Rechtsgeschiedenis, t. 46, 1978, p. 337.

42 J. Molinet J., Chroniques..., vol. 1, p. 36.

43 J. de LA Chauvelays, Les armées des trois premiers ducs de Bourgogne..., p. 325-326.

44 On trouvera un tableau résumant les différentes peines prévues par la législation militaire de Charles le Hardi dans B. SCHNERB, Un thème de recherche..., p. 106.

45 J. de LA Chauvelays, Les armées des trois premiers ducs de Bourgogne..., p. 325.

46 Ibid., p. 326-327.

47 F. Lot, L'art militaire et les armées au Moyen Âge, en Europe et dans le Proche-Orient, vol. 2, Paris, Payot, 1946, p. 116.

48 H. L. G. Guillaume, Histoire de l'organisation militaire..., p. 200-201. Sur l'entraînement comme moyen de contrôle, voir L. W. BURGENER, Les jeux et les exercices physiques en Suisse aux $X V^{e}$ et $X V I^{e}$ siècles, dans Éducation et hygiène du corps à travers l'histoire, éd. P. LÉVÊQUE, Dijon, Éditions de l'université de Dijon, 1991, p. 109-118 (Publications de l'Association interuniversitaire de l'Est, 25) ; J.-M. MEHL, Une éducation du corps à la fin du Moyen Âge et au début de la Renaissance : le tir à l'arc en France et en Angleterre, dans Idem, p. 17-27.

49 R. VAUGHAN, Charles the Bold..., p. 209.

50 W. H. MCNEILL, Drill/Marching, dans The Reader's Companion to Military History, éd. R. COWlEy et G. PARKER, New York, Houghton Mifflin, 1996, p. 142. 
Une oisiveté dangereuse. Vers une réglementation du "temps libre" dans les ar...

51 O. van Nimwegen, The Dutch Army and the Military Revolutions, 1588-1688, Woodbridge, The Boydell Press, 2010, p. 100-112.

52 C. Allmand, Did the De re militari of Vegetius influence the military ordinances of Charles the Bold?, dans Publications du centre européen d'études bourguignonnes, t. 41, 2001, p. 135-143; D. PAROTT, The Business of War : Military Enterprise and Military Revolution in Early Modern Europe, Cambridge, Cambridge University Press, 2012, p. 98.

53 G. Oestreich, Neostoicism and the Early Modern State,Cambridge, Cambridge University Press, 1992, VIII-280 p. (Cambridge studies in early modern history) ; P. SPIERENBURG, Protestant attitudes to violence : the early Dutch Republic, dans Crime, Histoire \& Sociétés t. 10, 2006, $\mathrm{n}^{\circ} 2$, p. 5-31.

54 Mémoires pour servir à l'histoire de France..., p. 287 ; C. Allmand, Did the De re militari..., p. 138.

$\underline{55}$ Mémoires pour servir à l'histoire de France..., p. 286.

56 C. Allmand, Did the De re militari..., p. 138.

57 L. Gollut, Les mémoires historiques..., col. 1256 ; C. Allmand, Did the De re militari..., p. 140. Sur la place du serment dans la construction idéologique de l'État bourguignon, voir J.-B. SANTAMARIA, Servir le prince et garder la loi de la ville. Les serments des officiers du duc de Bourgogne au sud des Pays-Bas (fin XIV $V^{e}$ milieu XV siècle), dans Histoire urbaine, t. 39, 2014, $\mathrm{n}^{\circ} 1$, p. 85-103.

58 Q. VERREYCKEN, Le soldat face au sacré. La lutte contre le viol des femmes et des lieux saints dans les armées de Charles le Hardi (1465-1477), moyen de promotion d'un nouveau modèle de comportement des gens de guerre ?, communication inédite au colloque Armée et religion, $X V^{e}$-XIX $X^{e}$ siècle, Nancy, Université de Lorraine, 21-22 octobre 2013 (remis pour publication).

59 H. L. G. Guillaume, Histoire de l'organisation militaire..., p. 199.

60 F. GODEFRoy, Dictionnaire de l'ancienne langue française et de tous ses dialectes du IX au XV $V^{e}$ siècle, vol. 6, Paris, F. Vieweg, 1890, p. 3-4, s. v. part.

61 M. Foucault, Sécurité, Territoire, Population. Cours au Collège de France. 1977-1978, Paris, Gallimard, 2004, p. 59 : « la normalisation disciplinaire consiste à poser d'abord un modèle, un modèle optimal qui est construit en fonction d'un certain résultat, et l'opération de la normalisation disciplinaire consiste à essayer de rendre les gens, les gestes, les actes conformes à ce modèle, le normal étant précisément ce qui est capable de se conformer à cette norme et l'anormal, ce qui n'en est pas capable ».

62 E. Goffman, Asiles : études sur la condition sociale des malades mentaux et autres reclus, trad. fr. de L. et C. LAINÉ, Paris, Éditions de Minuit, 1968, p. 41 (Le sens commun).

63 L. Gollut, Les mémoires historiques..., col. 1254.

64 H. L. G. Guillaume, Histoire de l'organisation militaire..., p. 201. 
65 B. SCHNERB, Le recrutement social et géographique...

66 C. GaUvard, La Fama, une parole fondatrice, dans Médiévales, t. 24, 1993, p. 5-13 ; A. Porteau-BitKer, A. TAlaZAC-LaURent, La renommée dans le droit pénal laïque du XIIIe au XVe siècle, dans Médiévales, t. 24, 1993, p. 67-80.

67 Ces chiffres diffèrent de ceux évoqués dans Q. VeRREYCKEN, « Pour nous servir en l'armée »..., p. 98-102 suite à de nouvelles investigations dans les registres et la découverte de lettres qui avaient alors échappé à notre vigilance.

68 ADN B $1695 \mathrm{f}^{\circ} 46 \mathrm{r}^{\circ}-\mathrm{v}^{\circ}$.

$\underline{69}$ Voir note 35 .

70 J. Molinet, Chroniques..., vol. 1, p. 61-62.

71 P. Contamine, L'armée de Charles le Téméraire : expression d'un État en devenir ou instrument d'un conquérant?, dans Aux armes, citoyens ! Conscription et armée de métier des Grecs à nos jours, éd. M. VAÏsSE, Paris, Colin, 1998, p. 73-74.

PDF généré automatiquement le 2020-07-07 12:19:20

Url de l'article : https://popups.uliege.be:443/1370-2262/index.php?id=297 